\title{
Conservative random walks in confining potentials
}

\author{
Bartłomiej Dybiec $^{\dagger}$, Karol Capała ${ }^{\dagger}$, Aleksei V. Chechkin ${ }^{\ddagger}, \sharp$, and \\ Ralf Metzler $\sharp$ \\ † Marian Smoluchowski Institute of Physics, and Mark Kac Center for Complex \\ Systems Research, Jagiellonian University, ul. St. Łojasiewicza 11, 30-348 Kraków, \\ Poland \\ $\ddagger$ Akhiezer Institute for Theoretical Physics NSC KIPT, Kharkov 61108, Ukraine \\ $\sharp$ Institute for Physics \& Astronomy, University of Potsdam, 14476 Potsdam-Golm, \\ Germany \\ E-mail: bartek@th.if.uj.edu.pl
}

\begin{abstract}
Lévy walks are continuous time random walks with spatio-temporal coupling of jump lengths and waiting times, often used to model superdiffusive spreading processes such as animals searching for food, tracer motion in weakly chaotic systems, or even the dynamics in quantum systems such as cold atoms. In the simplest version Lévy walks move with a finite speed. Here, we present an extension of the Lévy walk scenario for the case when external force fields influence the motion. The resulting motion is a combination of the response to the deterministic force acting on the particle, changing its velocity according to the principle of total energy conservation, and random velocity reversals governed by the distribution of waiting times. For the fact that the motion stays conservative, that is, on a constant energy surface, our scenario is fundamentally different from thermal motion in the same external potentials. In particular, we present results for the velocity and position distributions for single well potentials of different steepness. The observed dynamics with its continuous velocity changes enriches the theory of Lévy walk processes and will be of use in a variety of systems, for which the particles are externally confined.
\end{abstract}

\section{Introduction}

Already in 1795 Dutch physician Jan Ingenhousz observed irregular motion of coal dust particles on the surface of alcohol. Scottish botanist Robert Brown used more systematic studies of jittery motion of various inanimate materials following his observation of the same zigzagging of pollen granules extracted from pollen grains, in 1828 [1]. More systematic studies of diffusive particle motion are due to Gouy [2] and, in particular, Perrin, whose seminal 1908 paper [3] prompted a whole series of even more refined experiments. Two remarkable examples of the latter are the experiments of Nordlund [4] and Kappler [5] using moving film plates to produce long, individual time series of the motion. The theoretical analysis of the Brownian motion of thermally activated particles appeared at the start of the 20th century, mainly promoted by Smoluchowski [6], Einstein [7], and Langevin [8]. 
A standard way to define Brownian motion within the theory of stochastic processes is in terms of the Wiener process, which can be represented as the Langevin equation [9, 10]

$$
\frac{d x(t)}{d t}=\xi(t),
$$

where the Gaussian white noise $\xi(t)$ with zero mean $\langle\xi(t)\rangle=0$ and $\delta$-correlation $\left\langle\xi(t) \xi\left(t^{\prime}\right)\right\rangle=\delta\left(t-t^{\prime}\right)$ correspond to the increments of the Wiener process. The latter are independent and identically distributed according to the normal (Gaussian) distribution $x(t)-x(s) \sim \mathcal{N}(0, t-s)$. Physically, this is the natural consequence of the assumption that interactions of the test particle with its thermal environment occur on a faster time scale. Moreover they are independent and bounded. Therefore, the collisions with the bath particles can be approximated by Gaussian white noise. Note that here and in the following we adopted a dimensionless notation, in which position, time, and mass have unit dimension.

The Wiener process can be extended to $\alpha$-stable motions, for which the increments remain independent but are distributed according to $\alpha$-stable densities with heavy-tailed power-law asymptotics (Lévy flights) [11, 12. The Wiener process is included in the $\alpha$-stable processes in the limiting case $\alpha=2$. $\alpha$-stable processes can be extended to the case of external forces, resulting in the more general form of the Langevin equation

$$
\frac{d x(t)}{d t}=-V^{\prime}(x)+\xi(t),
$$

where $V(x)$ is the potential resulting in the deterministic force $F(x)=-V^{\prime}(x)$. Equation (2) provides a starting point for the analysis of a large variety of noise induced phenomena [13, 14, 15, 16]. We note that Lévy flights are often invoked as optimal search strategies, due to their fractal sample paths combining longer excursions with local search events [17. However in the presence of an external drift the advantage of Lévy flights over normal Brownian search may become significantly reduced or even turn into a disadvantage [18].

The stochastic equation (2) displays some non-physical properties. These are often negligible on the relevant space and time scales. However, from a conceptual point of view random walks generated by the scheme (2) involve an infinite propagation speed. For Brownian motion this shortcoming was particularly realised in the context of heat flow. There it was remedied by the introduction of the Cattaneo or telegrapher's equation on the level of the diffusion equation, leading to short-time ballistic motion with a finite horizon of propagation [19, 20, 21]. Even more so this problem arises for $\alpha$-stable noises with $\alpha<2$ : due to the significant probability of extremely long jumps the variance diverges [22, 23, 24]. A remedy for this infinite propagation speed can be introduced in terms of a spatio-temporal coupling of jump lengths and associated waiting times. This concept was introduced in the continuous time random walk scheme in the form of Lévy walks by Shlesinger and coworkers in 1982 [25], see also [26, 27].

In their simplest version Lévy walks couple jump lengths and the corresponding waiting time by a constant speed $v$. Random velocity changes occur after independent 
and identically distributed waiting times. These waiting times thus determine the flight time and thus the travelled distance in between velocity changes. Lévy walks were successfully applied to model the dynamics of tracer particles in weakly chaotic systems [28, 29, 30] and in the dynamics of cold atom systems [31]. A particular field of application of Lévy walks are random search processes, for instance, of animals searching for sparse food. Here Lévy walks combine the above-mentioned advantage of Lévy flights with a physically meaningful, finite variance of the motion [26, 32. Remarkably, Lévy walk search statistics were unveiled in intracellular motion driven by molecular motors [33, 34. We also note that Lévy walks exhibit ultraweak ergodicity breaking such that time and ensemble averaged observables merely differ by a constant, $\alpha$-dependent factor, and that they fulfil a linear response relation, among other interesting physical properties [35, 36, 37, 38, 39].

In the classical Lévy walk setup [27] no forces are acting on the moving particle. Therefore, its energy $\mathcal{E}$ is fixed to the kinetic energy $v^{2} / 2$, and is constant. At present, despite the wide use of the Lévy walk model a comprehensive conceptual understanding of the response of Lévy walks to an arbitrary external force field remains elusive. Here, we present a possible extension of the Lévy walk framework which takes into account such force fields. As in the classical Lévy walk model the system is considered to be conservative in the sense that the total energy is conserved, while the velocity is randomly reversed at time events defined by the waiting time distribution. This conservative scheme immediately implies that the Lévy walk speed is no longer constant but constantly varies along with the potential energy corresponding to a given position $x$. The resulting stationary distribution will therefore differ from the Boltzmann distribution, which emerges in a thermal system and allows for infinitely far yet exponentially suppressed excursions. The conservative model with well-defined maximum excursions due to the constant energy requirement will thus be more appropriate for cases when the particle is not allowed to cross a maximum distance while experiencing a restoring force. An example for such a behaviour could be the confinement of an animal searching for food while being limited to move only within their homing range. When approaching the border of the homing range the animal becomes more reluctant and thus slower. In such a scenario it may thus make sense that the velocity decreases with the distance from the centre of the system. More microscopically our conservative Lévy walk model may represent a molecular motor in a biological cell [33, 34 attempting to pull an anchored cargo. Indeed, with growing resisting force molecular motors achieve lower speeds and efficiencies [40, 41].

In the following section 2 we introduce our conservative Lévy walk model. Its properties are explored in detail in sections 3 and 4 . Finally we draw our conclusions in section 5 


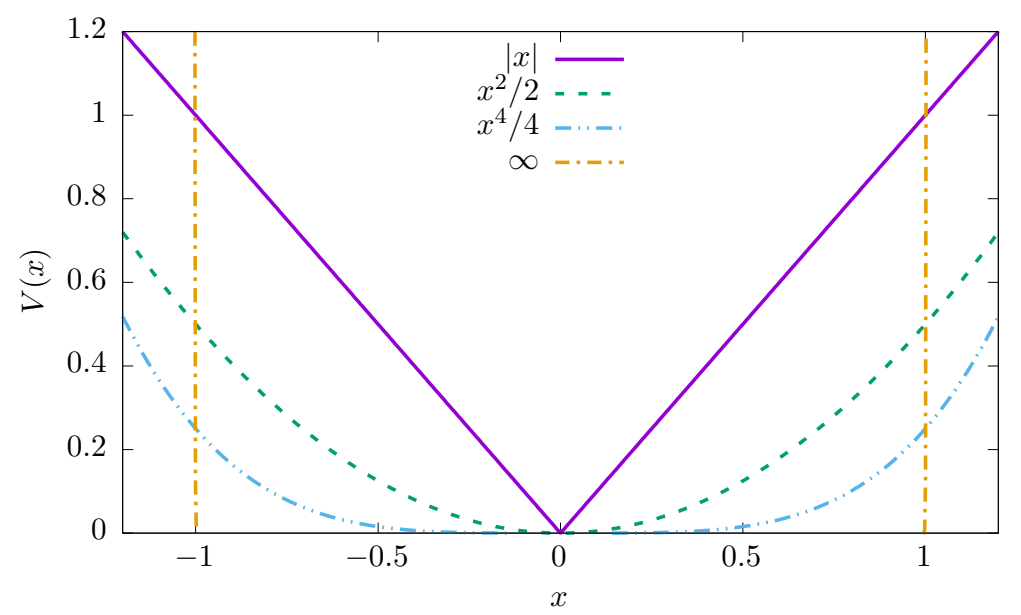

Figure 1. Single well potential $V(x)=|x|^{n} / n$ for $n=1,2,4$ as well as for $n=\infty$, which represents an infinite rectangular potential well.

\section{Conservative random walk model}

We start with the motion of a test particle described by the classical Newton equation

$$
\frac{d^{2} x(t)}{d t^{2}}=-V^{\prime}(x)
$$

in our dimensionless notation. For the external potential we choose the power-law form

$$
V(x)=\frac{|x|^{n}}{n}
$$

for which we consider the integer values $n=1,2,4$ and $n=\infty$, that is, a softerthan-harmonic, harmonic, and stronger-than-harmonic potential as well as a box with infinitely steep walls, see figure 1. The motion encoded by equation (3) for confining potentials of the form (4) is always periodic with the period

$$
T=4 \int_{0}^{x_{\max }} \frac{d x}{\sqrt{2[\mathcal{E}-V(x)]}} .
$$

Here $x_{\max }$ is the reversal point satisfying $V\left(x_{\max }\right)=\mathcal{E}$, and thus the period of the motion depends on the system energy $\mathcal{E}$, except for the harmonic case $n=2$, see reference [42]. The total energy $\mathcal{E}$ is determined by the initial condition $x(0)$ and $\dot{x}(0)$, or, more precisely $\mathcal{E}=\frac{1}{2} \dot{x}^{2}(0)+\frac{1}{n}|x(0)|^{n}$. In phase space, a particle described by relation (3) moves (clockwise) along the closed orbit determined by the system energy $\mathcal{E}$

$$
\mathcal{E}=\frac{1}{2} v^{2}+V(x)=\text { const }
$$

see figure 2. Naturally velocity reversals occur at the points of maximal distance from the origin $x_{\max }$. These reversals are soft in the sense that they occur at $v\left(x_{\max }\right)=0$.

In addition to the naturally occurring velocity reversals in the orbits of figure 2 we now introduce additional, random velocity reversals. In this scheme a particle moves according to equation (3) for a random time $\tau(\tau \geqslant 0)$ which is distributed according to the given density $\psi(\tau)$ of waiting times. With the probability $p=1$, the velocity 


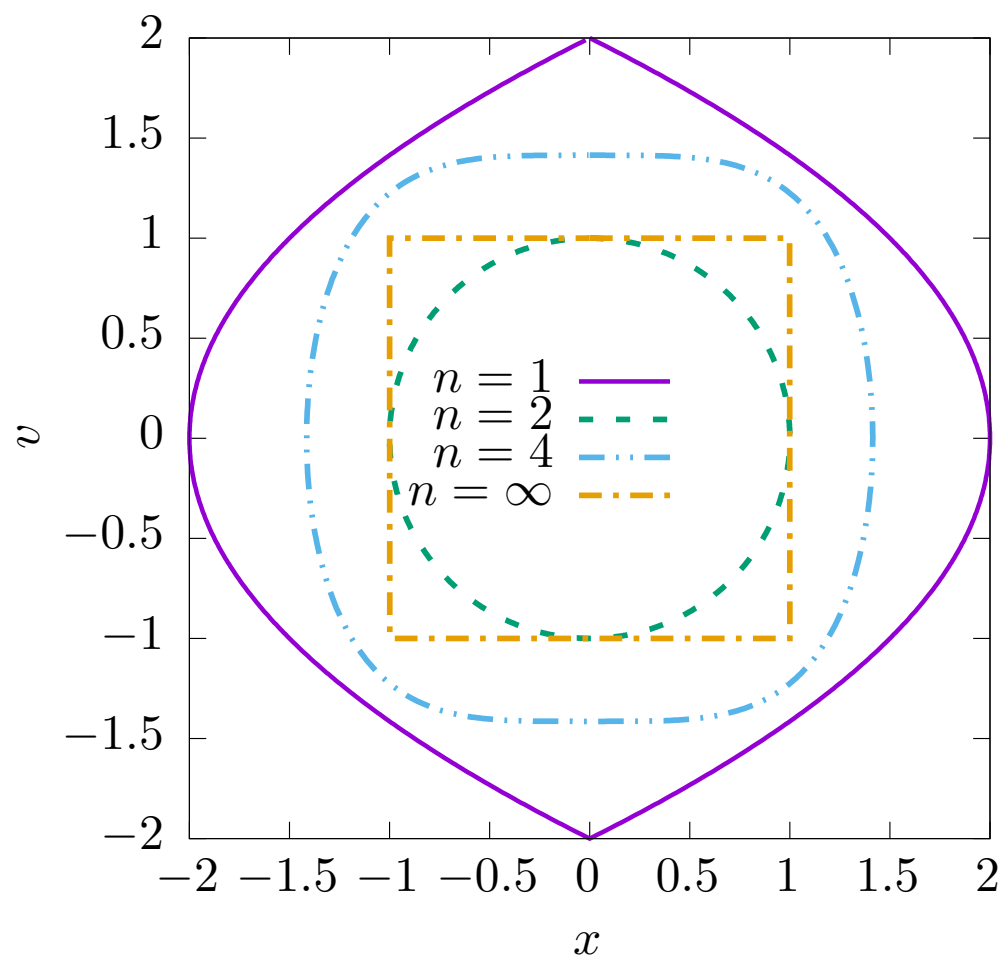

Figure 2. Phase space portrait of constant energy curves for $n=1,2,4$, and $\infty$. Note the soft velocity reversals at the points of maximal distance from the origin, where $v\left(x_{\max }\right)=0$.

is reversed, that is, $v(\tau) \rightarrow-v(\tau)$. These reversals are hard in the sense that they typically occur at points where $v(x) \neq 0$. Here, we assume that $\tau=|\zeta|$, where $\zeta$ follows a symmetric $\alpha$-stable density with characteristic function [11]

$$
\phi(k)=\langle\exp (i k \tau)\rangle=\exp \left(-\kappa^{\alpha}|k|^{\alpha}\right),
$$

where $\alpha(0<\alpha \leqslant 2)$ is the stability index and $\kappa(\kappa>0)$ is the scale parameter $¥$ For $\alpha<2, \psi(\tau)$ asymptotically behaves like the power-law

$$
\psi(\tau) \simeq \frac{1}{\tau^{\alpha+1}} .
$$

Consequently, for $\alpha<1$, the average between reversal time diverges. Additionally, we will consider the exponential waiting time distribution (Brownian creepers [44])

$$
\psi(\tau)=\lambda \exp (-\lambda \tau)
$$

with width $\lambda$. In this latter case moments of any order are finite.

After a hard velocity reversal a new waiting time $\tau$ is generated and the deterministic motion is continued until the next hard velocity reversal. If during the period $\tau$ a soft velocity reversal occurs at the points of maximal distance from the origin, the waiting time continues to be counted, that is, the waiting period in between two hard velocity reversals remains unaffected by soft reversals. Therefore, the system $\ddagger$ Note that we could have chosen a completely asymmetric, one-sided Lévy stable distribution instead. However, with the symmetric choice we can also consider $\alpha$ values larger than unity [11, 43]. 


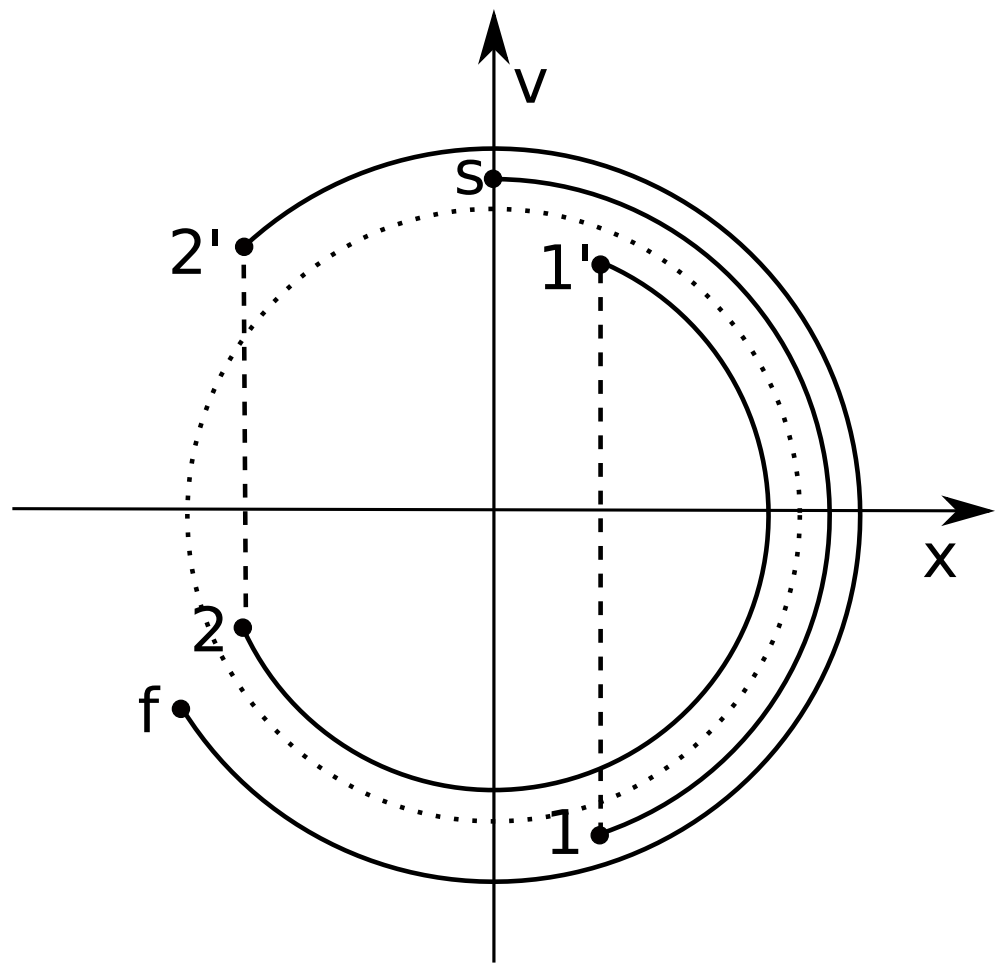

Figure 3. Schematic of the particle motion in phase space from starting point (s) to final point (f). Solid line shows a possible trajectory while dashed parts correspond to velocity reversals $\left(1 \rightarrow 1^{\prime}\right)$ and $\left(2 \rightarrow 2^{\prime}\right)$. The dotted circle represents $\mathcal{E}=$ const orbit on which a particle stays all the time, the different radii are only drawn for clarity.

evolves deterministically between the random velocity reversals. We expressly note that the velocity reversals only change the sign of the velocity thus keeping the system conservative with constant energy (6). We here assume each waiting time event leads to a hard velocity reversal with unit probability $\{$ In phase space the particle makes a jump from the point $(x, v)$ to the point $(x,-v)$ on the orbit determined by (6), without change in the direction of motion along the orbit, see figures 3 and 4

\section{Trajectories and mapping onto position and velocity distributions}

Figure 3 schematically depicts the motion of a particle described by equation (3) along with its velocity reversals in phase space. Due to energy conservation (clock wise) motion takes place on the closed $\mathcal{E}=$ const orbit, which is depicted by the dotted line. Note that for clarity the solid line representing the sample trajectory is drawn with a different radius. The points labelled $\mathrm{s}$ and $\mathrm{f}$ indicate the starting and final positions respectively. Points 1 and $1^{\prime}$ as well as 2 and $2^{\prime}$, connected with dashed lines, illustrate the hard velocity reversals. During these reversals a particle changes its velocity from unprimed to primed numbers, while the position remains the same. Figure 4 demonstrates some sample time evolutions of the velocity $v(t)$ (left column) and position $x(t)$ (right column)

$\S$ We could also assume that velocity reversals occur with probability $\frac{1}{2}$. 

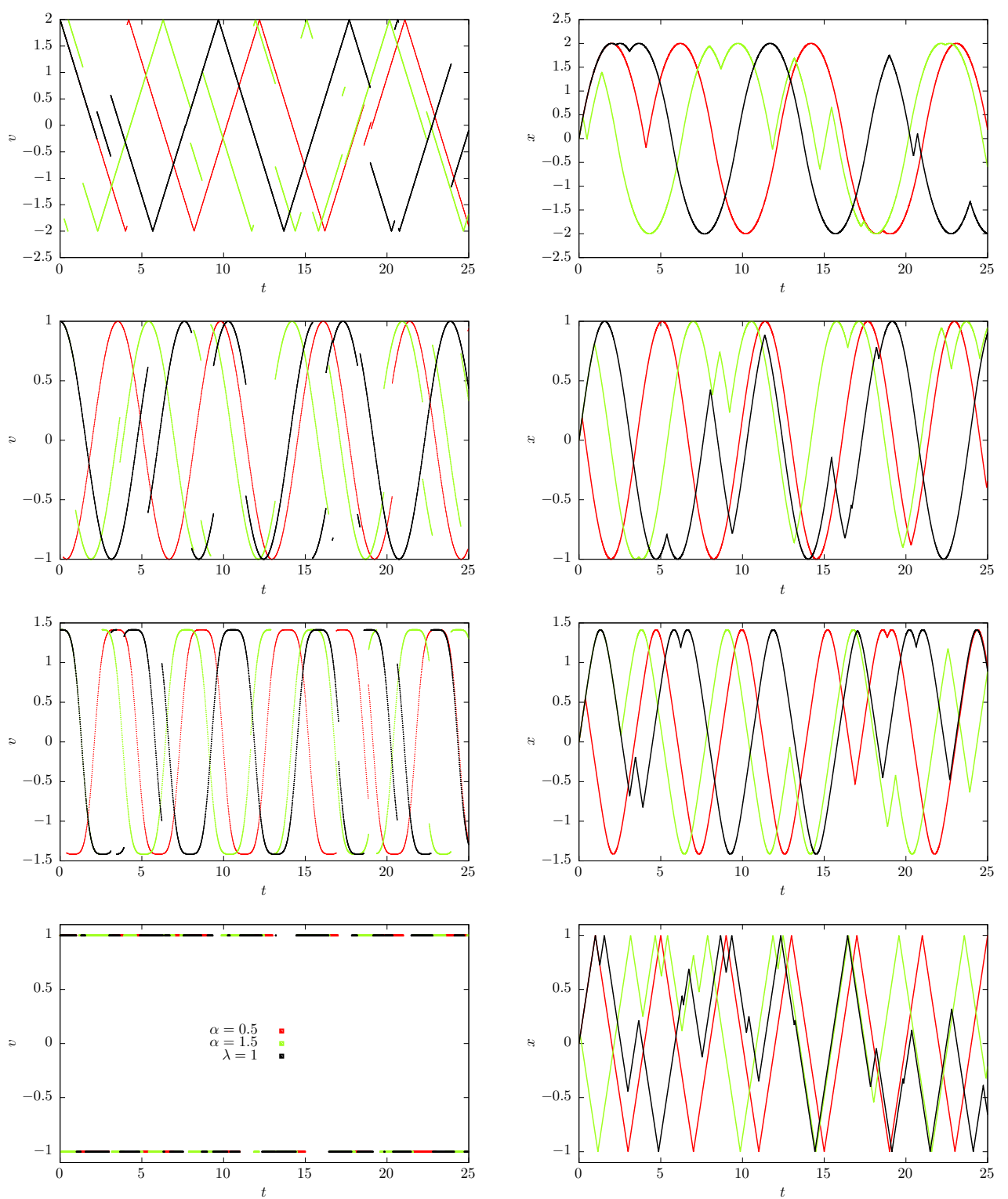

Figure 4. Sample trajectories for $n=1,2,4$, and $\infty$ (top to bottom). The different colours correspond to $\alpha=0.5$ (red), $\alpha=1.5$ (green), and the exponential case with the scale factor $\lambda=1$ (black), see bottom left panel for the legend. In the left column we show the velocity $v(t)$, the right column depicts the position $x(t)$. The simulations are described in section 4 below.

of a test particle for various model parameters and potential types.

Every hard velocity reversal is associated with advancing the solution $x(t)$ of equation (3) by a shift $\delta$

$$
\delta=\left\{\begin{array}{lll}
2 \mathcal{T}\left(|x(t)|, x_{\max }\right) & \text { if } & x(t) \times v(t)>0 \\
T-2 \mathcal{T}\left(|x(t)|, x_{\max }\right) & \text { if } & x(t) \times v(t)<0
\end{array},\right.
$$

where $\mathcal{T}\left(|x(t)|, x_{\max }\right)$ is the time needed to travel from $|x(t)|$ to the reversal point $x_{\max }$,

$$
\mathcal{T}\left(|x(t)|, x_{\max }\right)=\int_{|x(t)|}^{x_{\max }} \frac{d x}{\sqrt{2[\mathcal{E}-V(x)]}} .
$$




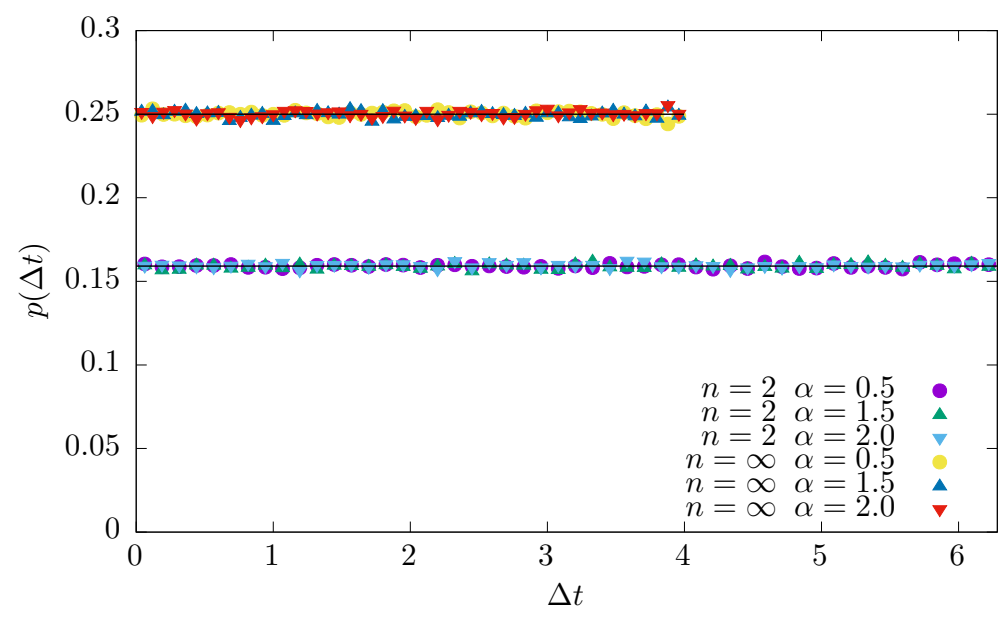

Figure 5. Accumulated time shift distribution $p(\Delta t)$, see equation 12 , for $n=2$ and $n=\infty$ with various values of $\alpha$. The solid lines represent $1 / 2 \pi$ and $1 / 4$, which are uniform densities over $[0, T]$ for $n=2(T=2 \pi)$ and $n=\infty(T=4)$.

$T$ is the period of the motion given in relation (5). The time shift (10) assures that $x(t)=$ $x(t+\delta)$ and $v(t)=-v(t+\delta)$, which in turn guarantee energy conservation. Numerical simulations of the model confirm that at sufficiently long time $t$ the accumulated time shift

$$
\Delta t=\sum_{i=0}^{N} \delta_{i} \bmod T
$$

is uniformly distributed on the interval $[0, T)$. The upper summation bound $N$ is defined by the condition

$$
\sum_{i=0}^{N-1} \tau_{i} \leqslant t \leqslant \sum_{i=0}^{N} \tau_{i}
$$

where the $\tau_{i}$ are the independent identically distributed random waiting times defined by the distribution (8). The observation that $\Delta t$ in expression (12) is uniform, $U([0, T)$ ), is shown in figure 5 for $n=2$ and $n=\infty$.

Due to the velocity reversals the phase space co-ordinates $x(t)$ and $v(t)$ are no longer deterministic but they become random variables. Moreover, position $x$ and velocity $v$ are not independent but are linked by the energy conservation constraint (6). Therefore, the velocity density $p(v)$ can be used to calculate the position distribution $p(x)$ through

$$
p(x)=\frac{|x|^{n-1}}{\sqrt{2[\mathcal{E}-V(x)]}} \times p(v= \pm \sqrt{2[\mathcal{E}-V(x)]}) .
$$

Analogously, $p(v)$ can be obtained from $p(x)$ through

$$
\begin{aligned}
p(v)= & {\left[n\left(\mathcal{E}-v^{2} / 2\right)\right]^{1 / n-1}|v| } \\
& \times p\left(x= \pm\left[n\left(\mathcal{E}-v^{2} / 2\right)\right]^{1 / n}\right) .
\end{aligned}
$$

In figure A1 we demonstrate that on top of the soft velocity reversals at the zerovelocity points, even for identical initial conditions hard reversals still maintain the 
random nature of the conservative Lévy walk motion. Consequently, after a finite time trajectories corresponding to the same initial conditions are becoming disparate, thus randomizing velocity $v(t)$ and position $x(t)$.

The cases $n=1$ and $n=2$ are fully traceable analytically. For $n \geqslant 3$ one has to rely on numerical methods. Consequently, we start our considerations with the case $n=2$ corresponding to the conservative Lévy walk harmonic oscillator. Consecutively, we proceed with the case $n=1$ and, finally, we consider the cases $n=4$ and $n=\infty$ numerically.

In the specific $n=2$ case the relation (3) describes the harmonic oscillator, for which

$$
x(t)=x_{\max } \sin (t+\varphi),
$$

where $\varphi$ is the initial phase. Without loss of generality it can be assumed that $\varphi=0$, i.e., $x(0)=0$ and $v(0)=v_{\max }$. The first velocity reversal at $t_{1}$ introduces the extra phase shift $\delta$ to equation (16), which is equal to

$$
\delta=\pi-2 t_{1} .
$$

The phase shift can be calculated from the condition

$$
\sin \left(t_{1}\right)=\sin \left(t_{1}+\delta\right)
$$

which is fulfilled for $\delta=0$ or $\delta=\pi-2 t_{1}$, as $\sin (u)=\sin (w)$ has two series of main solutions, $u=w$ and $u=\pi-w$. Only the latter solution assures that $v\left(t_{1}\right)=-v\left(t_{1}\right)$. Finally, for $n=2$ the phase shift fulfils the recursion

$$
\left\{\begin{array}{l}
\delta_{0}=0 \\
\delta_{n}=\pi-2 t_{n}-\delta_{n-1}
\end{array},\right.
$$

where $t_{n}$ are time instants of velocity reversal. These time instants are given by

$$
t_{n}=t_{n-1}+\tau
$$

with $t_{0}=0$.

For $\alpha>1$ (or for the exponential density) when the mean time $\tau$ between reversals is finite, from numerical simulations we conclude that at sufficiently long times $\Delta t$, see

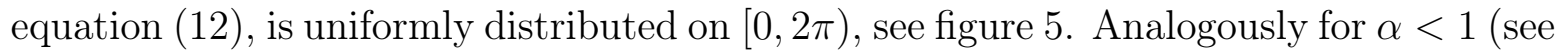
below) the distribution $p(\Delta t)$ also becomes uniform, however, due to the long memory of the initial conditions a significantly longer time is required as compared to the case $\alpha>1$. Therefore, both velocity and position are distributed according to the arcsine laws

$$
p(v)=\frac{1}{\pi \sqrt{1-v^{2}}}
$$

and

$$
p(x)=\frac{1}{\pi \sqrt{1-x^{2}}},
$$


where we interpret velocity and position as $v(t) / v_{\max }$ and $x(t) / x_{\max }$. Above results follow from the transformation of variables

$$
v(t) / v_{\max }=\cos (t+\delta \bmod 2 \pi),
$$

and

$$
x(t) / x_{\max }=\sin (t+\delta \bmod 2 \pi),
$$

where $(t+\delta \bmod 2 \pi)$ is uniformly distributed on $[0,2 \pi)$, that is, $U([0,2 \pi))$. This behaviour is indeed corroborated by our numerical analysis, see below.

A different situation occurs for $\alpha<1$, when the mean waiting time between hard velocity reversals diverges. In this situation, even at appreciably long times discrete slowly decaying peaks appear in the distributions $p(x)$ and $p(v)$, see [45]. These correspond to deterministic motion events without hard velocity reversal, that is, to the solution of equation (3) with below initial conditions (27) and (28). At the time instant when $p(x)$ and $p(v)$ are evaluated, the trajectories corresponding to the peaks will be at exactly the same position with exactly the same velocity. With increasing time the height of these peaks decreases as the likelihood for hard velocity reversals increases. Finally, in the limit $t \rightarrow \infty$, in analogy to the case $\alpha>1$, the peaks also disappear. Of course, these peaks can be completely eliminated by taking a random initial condition on the constant energy orbit.

A similar effect is observed for $V(x)=|x|$, in this case $v(t)$ is piecewise linear and $x(t)$ is piecewise parabolic. Assuming, analogously to the harmonic oscillator, that the velocity reversal introduces an extra phase shift to $x(t)$ and $v(t)$ leading to uniform distribution of $(t+\delta \bmod T)$ on $[0, T)$, transformation of variables shows that the velocity is uniformly distributed over $\left[-v_{\max }, v_{\max }\right]$,

$$
p(v)=\frac{1}{2 v_{\max }} .
$$

Furthermore, using relations (14) and (25) the density $p(x)$ can be calculated as

$$
p(x)=\frac{1}{4 \sqrt{\mathcal{E}(\mathcal{E}-|x|)}},
$$

where $x \in\left[-x_{\max }, x_{\max }\right]=[-\mathcal{E}, \mathcal{E}]$. Analogously, expression 26 can be calculated using the fact that $x(t)$ is piecewise parabolic. Our numerical results thus confirm the above assumption of the uniform distribution of $(t+\delta \bmod T)$.

\section{Numerical analysis}

As the motion in our conservative phase space is restricted to the total energy surface $\mathcal{E}=\frac{1}{2} v^{2}+V(x)=$ const the velocity $v(t)$ changes between the maximal values $-v_{\max }$ and $v_{\max }$. Similarly, the position $x(t)$ is restricted to $\left[-x_{\max }, x_{\max }\right]$, in contrast to thermally activated motion which can reach arbitrary values of the phase space co-ordinates, with the respective Boltzmann weights. For clarity of presentation the initial conditions are chosen such that the numerical values of $x_{\max }$ and $v_{\max }$ are identical and the resulting 


\begin{tabular}{c|c}
\hline$n$ & $T$ \\
\hline 1 & 8 \\
2 & $2 \pi \approx 6.283$ \\
4 & $\sqrt{\pi} \Gamma\left[\frac{5}{4}\right] / \Gamma\left[\frac{3}{4}\right] \approx 5.244$ \\
$\infty$ & 4 \\
\hline
\end{tabular}

Table 1. Periods $T$ of the periodic motion in potentials $V(x)=|x|^{n} / n$, see relation (5), for various values of the power $n$, with initial conditions (27) and 28).

$p(x)$ and $p(v)$, for fixed $n$, densities have the same support. For our case of unit mass and $n \neq 2$, the condition $x_{\max }=v_{\max }$ uniquely determines the total energy $\mathcal{E}$, which in turn can be used to calculate $x_{\max }$ and $v_{\max }$. For $n=2$ the closed orbit is a circle, and thus the relation $x_{\max }=v_{\max }$ always holds. As the initial condition any point on the $\mathcal{E}=$ const orbit can be chosen - we note that some of the observed results are sensitive to the initial conditions, as discussed below. We made the explicit choice

$$
x(0)=0
$$

and

$$
v(0)=\left\{\begin{array}{ll}
(2 / n)^{1 /(2-n)} & \text { for } n \neq 2 \\
1 & \text { for } n=2
\end{array} .\right.
$$

Different initial conditions will introduce an initial phase shift to the solutions $x(t)$ and $v(t)$. In principle, we would expect that for the case $\alpha>1$ fast mixing of the system occurs such that the long time properties are independent of the initial conditions. For $0<\alpha<1$, however, when the mean waiting time $\langle\tau\rangle$ diverges, the initial conditions should be visibly more persistent. In our finite-time simulations, we indeed observe a fast decay of the initial peaks in the position and velocity distributions for $\alpha>1$, while for $0<\alpha<1$ they are slower. Yet we do not see a clear change of the generic behaviour when $\alpha$ crosses the value of unity, see figures 6, 8, 9, and 10 .

As evidenced in figure A2 this decay is actually of power-law form whose scaling exponent depends on the exponent of the waiting time density. It is tempting to assume that the decay of the peak height is directly linked to the scaling exponent $\alpha$ of the waiting time density, in the spirit of "The single big jump principle" [46]. However, the results listed in table A1 appear not fully conclusive. For random initial conditions on the constant energy orbit, in contrast to fixed initial conditions, even for $\alpha<1$ there are no additional peaks in the densities $p(x)$ and $p(v)$ as the peaks are washed out.

For the general potential (4) with exponent $n$ the period of the deterministic motion is given by expression (5). The values of the periods $T$ corresponding to different $n$ with initial conditions 27) and (28) (or, more precisely, with the total energy $\mathcal{E}$ determined by the initial conditions) are listed in table 1 . For $n=4$, these values were used to obtain $p(x)$. 

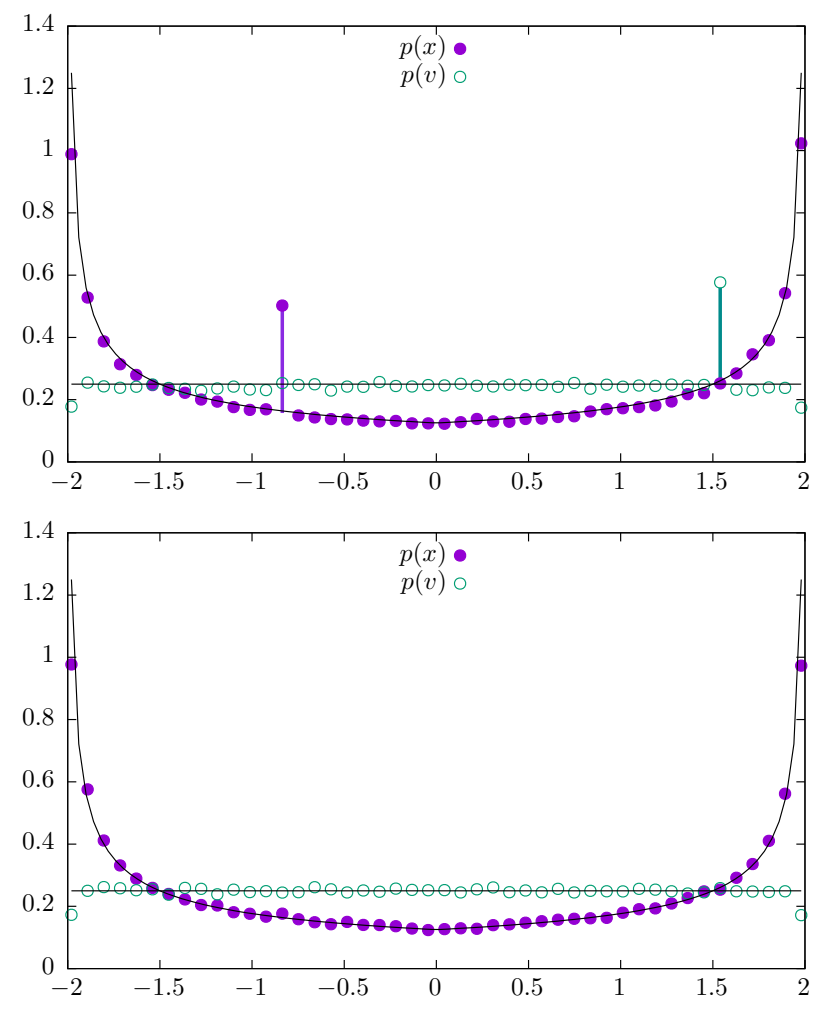

Figure 6. Probability densities $p\left(x, t=10^{3}\right)$ and $p\left(v, t=10^{3}\right)$ for $V(x)=|x|$ with $\alpha=0.5$ (top) and $\alpha=1.5$ (bottom). Solid lines are given by equations (25) and (26). The initial conditions are adjusted in such a way that $v_{\max }=x_{\max }$, see relations (27) and 28).

\begin{tabular}{c|c|c}
\hline$n$ & $\sigma(x)$ & $\sigma(v)$ \\
\hline 1 & $\sqrt{32 / 15} \approx 1.461$ & $\sqrt{4 / 3} \approx 1.155$ \\
2 & $\sqrt{1 / 2} \approx 0.707$ & $\sqrt{1 / 2} \approx 0.707$ \\
4 & 0.956 & 1.155 \\
$\infty$ & $\sqrt{1 / 3} \approx 0.577$ & 1 \\
\hline
\end{tabular}

Table 2. Asymptotic values of the standard deviations $\sigma(x)$ and $\sigma(v)$ of the position and velocity co-ordinates for periodic motion in the potentials (4), for various values of $n$ and initial conditions (27) and (28).

In the numerical evaluation for the case $n=4$ with Wolfram Mathematica equation (3) was used to construct the mapping $[0, T) \ni t \mapsto x(t)$. Then, using the constructed map and the assumption that $(t+\delta \bmod T)$ is uniform on $[0, T)$ the probability density $p(x)$ was obtained numerically by transformation of variables. In the final step, $p(x)$ was transformed into $p(v)$ using relation (15). With increasing steepness $n$ the potential wells become almost flat in the vicinity of the origin. This implies that in that region there is practically no external force acting on the test particle. Consequently, close to the origin for large $n$ the velocity is practically constant, see the third panel from the top in figure 4 . 

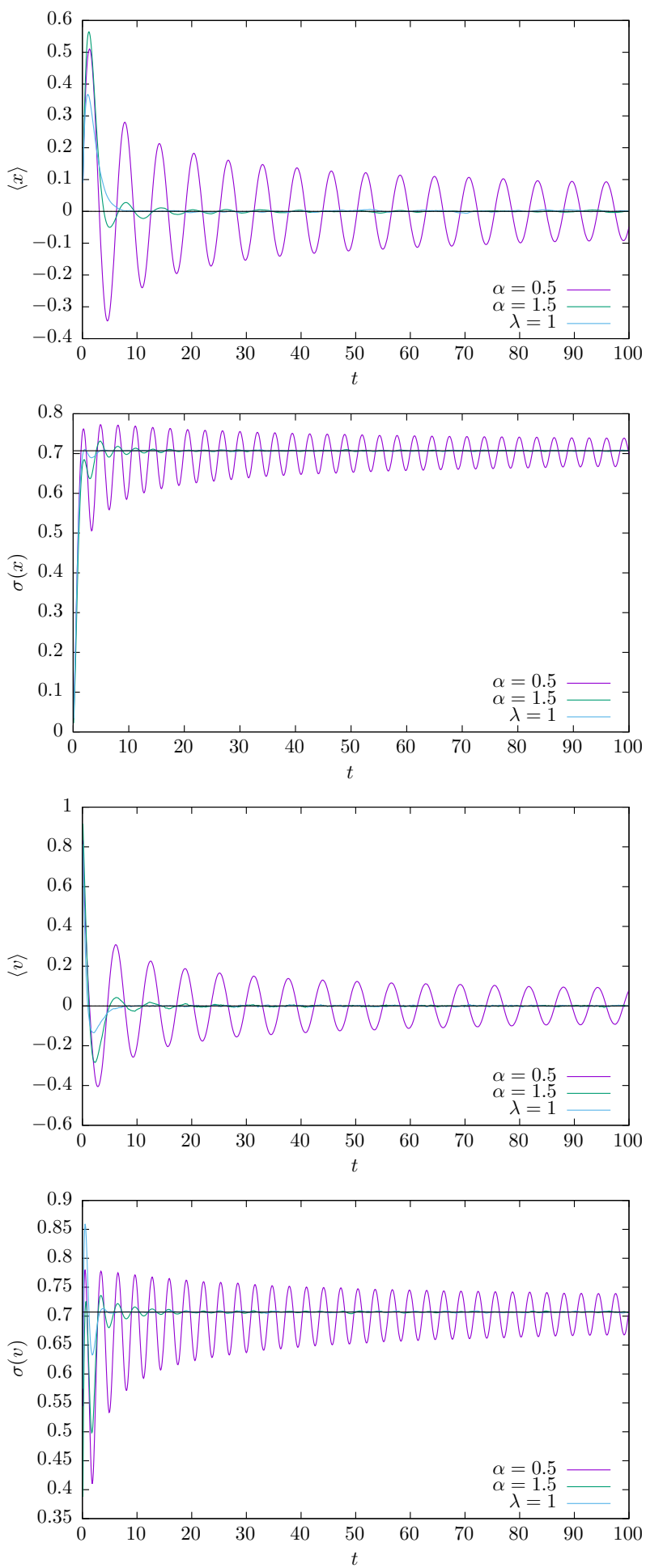

Figure 7. Case $n=2$, from top to bottom: mean position $\langle x(t)\rangle$, standard deviation $\sigma(x(t))$ of the position co-ordinate, mean velocity $\langle v(t)\rangle$, and standard deviation $\sigma(v(t))$ of the velocity. Solid lines represent the theoretical values of the averages and standard deviations given in table 2 The initial conditions (27) and (28) assure that $v_{\max }=x_{\max }$. 

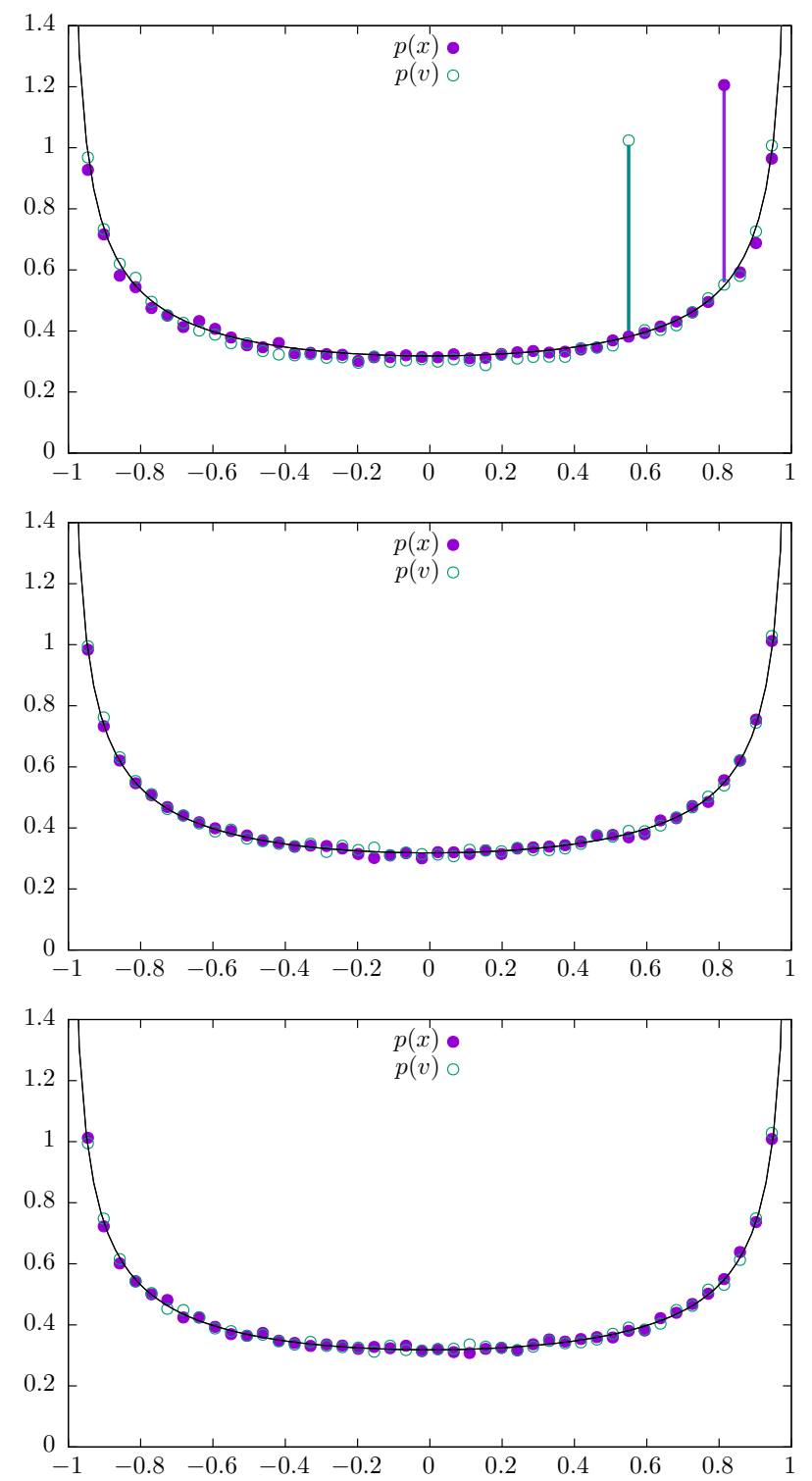

Figure 8. Probability densities $p\left(x, t=10^{3}\right)$ and $p\left(v, t=10^{3}\right)$ for $V(x)=x^{2} / 2$ with $\alpha=0.5$ (top) and $\alpha=1.5$ (middle). We also show the case of an exponential waiting time density with the scale factor $\lambda=1$ (bottom). Solid lines correspond to the values in equations 21 and 22 .

Therefore, the procedure based on the $[0, T) \ni t \mapsto v(t)$ mapping cannot be successfully applied to determine $p(v)$ when $x(t) \approx 0$. To calculate the density $p(v)$ a transformation of variables $x \rightarrow v$ has to be used, see equation (15). Nevertheless, despite being more robust than $[0, T) \ni t \mapsto v(t)$ mapping, even the numerical transformation of variables results in some numerical instabilities, see figure 9 .

The densities $p(x)$ and $p(v)$

The conservative random walk model based on equations (3) and (4) as well as random, hard velocity reversals was studied by Monte Carlo simulations. Multiple realisations 

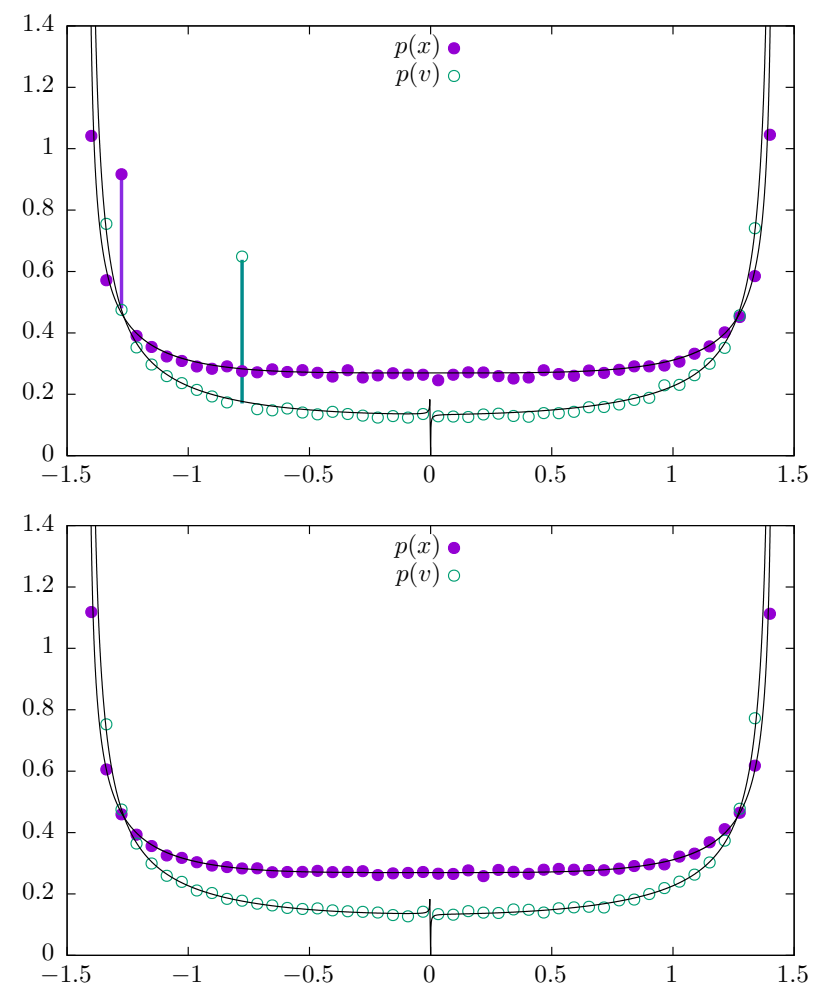

Figure 9. Probability densities $p\left(x, t=10^{3}\right)$ and $p\left(v, t=10^{3}\right)$ for $V(x)=x^{4} / 4$ with $\alpha=0.5$ (top) and $\alpha=1.5$ (bottom). The solid lines are calculated using Wolfram Mathematica.
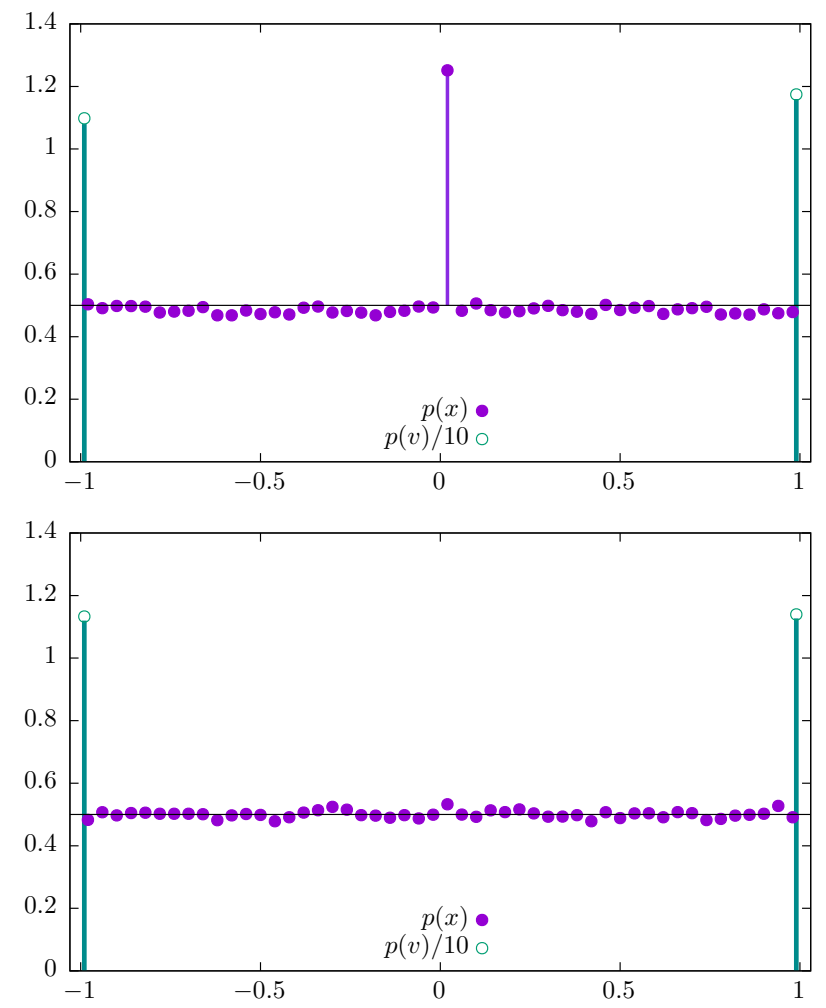

Figure 10. Probability density $p\left(x, t=10^{3}\right)$ and $p\left(v, t=10^{3}\right)$ for infinite rectangular potential well with $\alpha=0.5$ (top panel) and $\alpha=1.5$ (bottom panel). 
were simulated with the velocity Verlet algorithm [47]. Individual sample trajectories of finite length are displayed in figure 4 for various systems parameters. In particular, for finite time $t$, we show the difference between power-law waiting times with finite and infinite mean waiting time, with exponential waiting times.

From the ensemble of realisations the densities $p(x)$ and $p(v)$ were constructed and compared with the theoretical predictions. Figure 7 shows the oscillatory dynamics with a converging envelope of the mean values and standard deviation of the position $x(t)$ and the velocity $v(t)$ for the harmonic potential, $n=2$. Due to the symmetry of the setup, even for an asymmetric initial condition the densities for position and velocity converge to symmetric forms. Therefore the average values asymptotically tend to zero. As shown in figure A2 the decay of the envelope of this dynamics is of power-law form. The standard deviations converge to their asymptotic values given in table 2. For diverging mean waiting time for hard velocity reversals corresponding to $0<\alpha<1$, clear periodicities in the mean and standard deviations of position and velocity are visible. Nevertheless, as discussed in Appendix A, the amplitude of the periodicity decays in time with a characteristic time scale depending on the value of the exponent $\alpha$, see figure A2 and table A1. The observed periodicity agrees with the period of the motion for $\langle x(t)\rangle$ and $\langle v(t)\rangle$, as provided in table 11. The time dependencies of $\sigma(x(t))$ and $\sigma(v(t))$ are characterised by the half periods as given in table 1$\}\left\langle x^{2}(t)\right\rangle$ and $\left\langle v^{2}(t)\right\rangle$ indeed feature half the periodicity of $\langle x(t)\rangle$ and $\langle v(t)\rangle$. Table 2 provides the asymptotic values of the standard deviations. For $n=1, n=2$ and $n=\infty$ they are calculated exactly from the theoretical densities $p(x)$ and $p(v)$, while for $n=4$ they were obtained numerically from $p(x)$ and $p(v)$.

Figures 6, 8, 9, and 10 display the finite time distributions $p(x, t)$ and $p(v, t)$ with

$\alpha=0.5, \alpha=1.5$ and $\kappa=\sqrt{2}$ as well as $\lambda=1$ (exponential waiting times), respectively, for increasing values of $n$ at $t=10^{3}$. Localised peaks in $p(x)$ and $p(v)$ for $\alpha=0.5$ are located at values of $x(t)$ and $v(t)$ corresponding to the deterministic motion, see equation (3). The results for the exponential waiting time distribution $(\lambda=1)$ are analogous to the case with finite mean waiting time corresponding to $1<\alpha \leqslant 2$. Therefore, we conclude that the model properties are not very sensitive to the exact shape of the waiting time distribution. The finiteness or infinity of the mean waiting time for $1<\alpha<2$ and $0<\alpha<1$, respectively, appears to only set the rate of convergence to the stationary state.

The solid lines in figures 6, 8, 9, and 10 represent the theoretical curves given by relations (21) and (22) as well as (25) and (26). For the case $n=4$ they are calculated with Wolfram Mathematica. As mentioned above, the initial conditions are adjusted such that $v_{\max }=x_{\max }$, as given by equations 27) and (28). Some numerical instabilities are visible in figures 9 where the distribution $p(v)$, calculated from $p(x)$, fluctuates around the zero line. Small velocities are recorded when $x \approx \pm x_{\max }$. In this region the numerical inversion of $x(t)$ introduces some error, which is responsible for the $p(v)$ fluctuations.

In the limit of $n \rightarrow \infty$ the potential $V(x)=|x|^{n} / n$ becomes comparable to the 


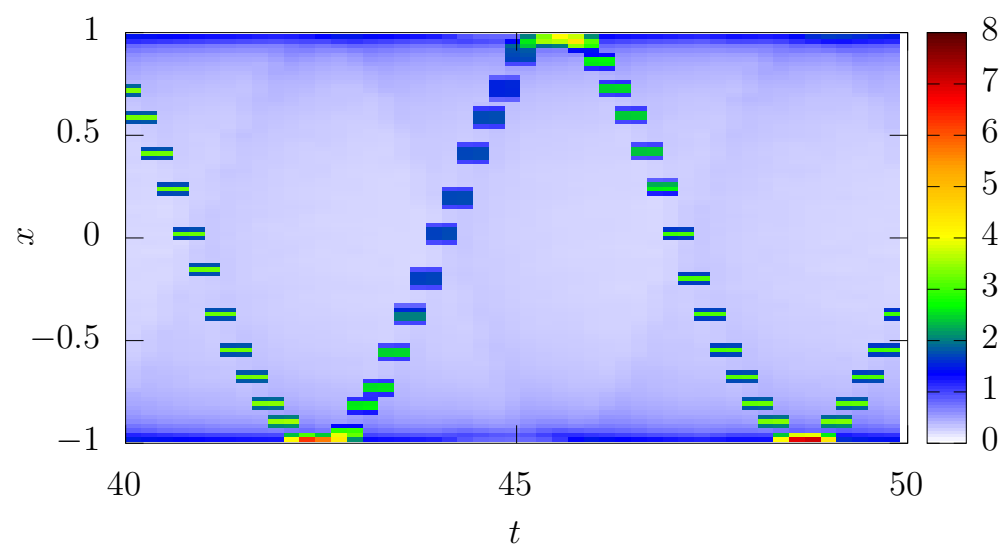

Figure 11. Sample time dependent probability density $p(x, t)$ for $\alpha=0.5, n=2$, and initial conditions (27) and (28) The motion appears almost fully deterministic due to lack of hard velocity reversals. See also the discussion in section 5 .

infinite deep rectangular potential well, see figure 1. In this case $p(x)$ is uniform and the formula for $p(v)$ reads

$$
p(v)=\frac{1}{2}\left[\delta\left(v-v_{0}\right)+\delta\left(v+v_{0}\right)\right],
$$

which is nicely corroborated in figure 10 . Note that $v_{0}$ is set to $v_{0}=1$ and $x_{\max }$ is set to $x_{\max }=1$. For $0<\alpha<1$ isolated persistent peaks in the densities $p(x)$ and $p(v)$ are visible, see figure 10 .

\section{Tails versus central parts of waiting time distributions}

Figure 11 presents the sample time dependent probability density $p(x, t)$ as a heatmap. The presented results correspond to $\alpha=0.5, n=2$ for initial conditions (27) and (28). As this case is part of our discussion for diverging mean waiting time, the dominating slowly disappearing maxima of $p(x, t)$ correspond to the deterministic motion $x(t)$ without hard velocity reversals. The projection of the maxima is thus given by $\sin (t)$, the solution of equation (3) for the selected setup. The maximum value of $p(x, t)$ decays with time due to hard velocity reversals, which eventually will occur. The decay rate is determined by $\alpha$ in the sense that larger values of $\alpha$ lead to more frequent randomising hard velocity reversals. Note the faint shadow line of the sine function shifted by half a period, $\delta=\pi$. Its origin is discussed in relation to figure 12. The case of $\alpha<1$ when the decay of the peaks is slow, should be contrasted with the case $\alpha>1$ : namely, when the mean time between velocity reversals is finite, the disappearance of the maxima of $p(x, t)$ is fast.

An interesting dependence is observed for even smaller values of the stability index $\alpha$, see top panel of figure 12 . For $\alpha=0.3$ and up to intermediate times the distributions $p(x)$ and $p(v)$ exhibit two peaks. The higher dominating peak corresponds to deterministic motion without hard velocity reversals, which occur with appreciable probability due to the scale-free nature of the waiting time distribution. The lower, 

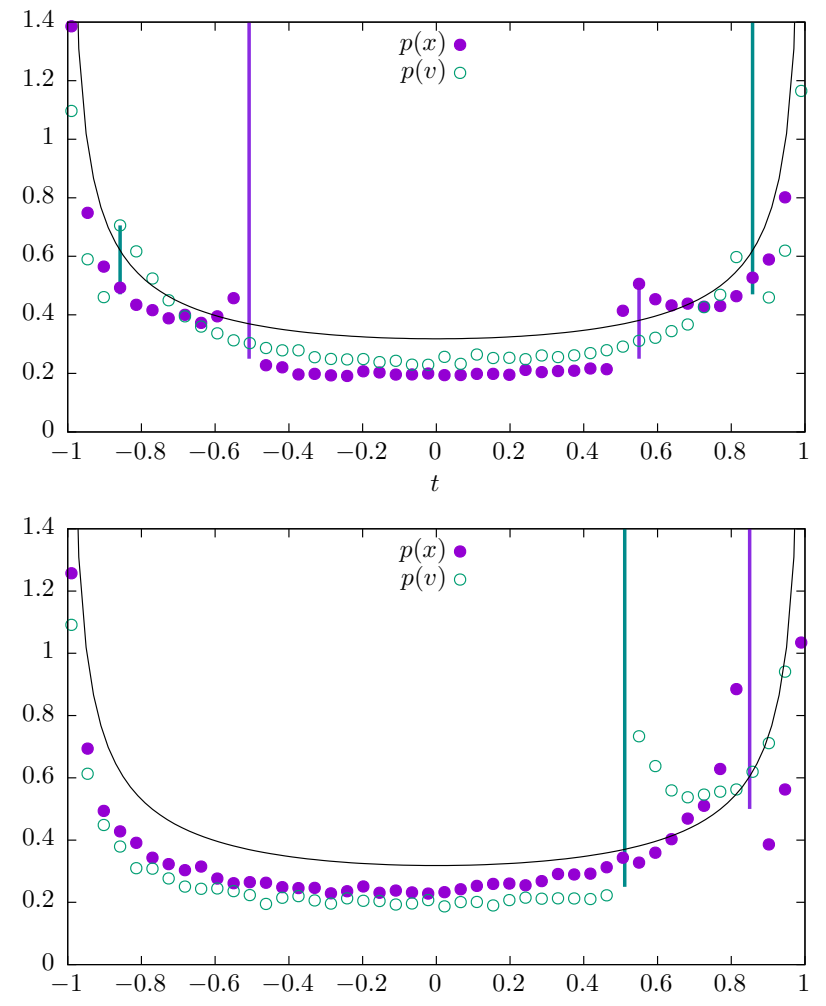

Figure 12. Distributions of $p(x, t=100)$ and $p(v, t=100)$ for $\alpha=0.3$ with $n=2$ for the initial condition given by Eqs. (27) and (28) (top panel) and $v(0)=0$ with $x(0)=x_{\max }($ bottom panel) .

symmetrically localised peak corresponds to those trajectories which were immediately reversed. A significant fraction of those reversed trajectories continues deterministically, thus giving rise to the secondary transient peak in position and velocity distributions. With increasing time the height of the peaks is decreasing and finally the system reaches its stationary density. The height of the secondary peak is decaying relatively fast with the stability index $\alpha$. A relatively faint reminiscence of the secondary peak can also be observed for stability index $\alpha=0.5$ in figure 11. These secondary peaks arise due to immediately reversed trajectories are confirmed by the cumulative density of waiting times, see figure 14, which shows that for small $\alpha$ there is a significant probability of (immediate) reversals.

Initial conditions (27) and (28) represent the situation when the particle motion is started in the minimum of the potential with the non-zero initial velocity. In the course of time velocity decreases as the potential energy grows. Finally, when $x=x_{\max }$ the velocity becomes equal to 0 and a soft reversal takes place. Such an initial condition results in the maximal initial velocity. Consequently, an immediate hard velocity reversal is well visible and it results in the appearance of secondary peaks in position and velocity distributions, see top panel of figure 12 . In contrast to (27) and (28) one can assume that a motion is started at $x=x_{\max }$ with zero initial velocity. On the one hand, both initial conditions result in the motion along the same orbit. On the other hand, zero initial 

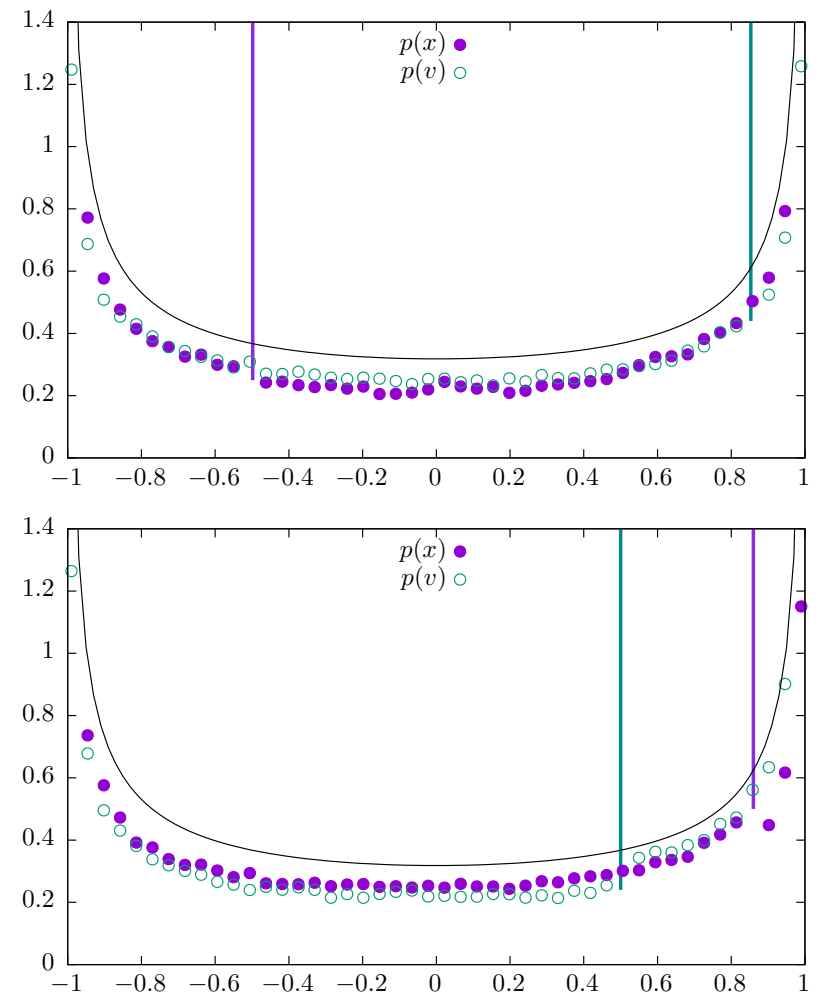

Figure 13. Distributions of $p(x, t=100)$ and $p(v, t=100)$ for Pareto distributions with $\alpha=0.3$ and $n=2$ for the initial condition given by Eqs. (27) and 28) (top panel) and $v(0)=0$ with $x(0)=x_{\max }$ (bottom panel).
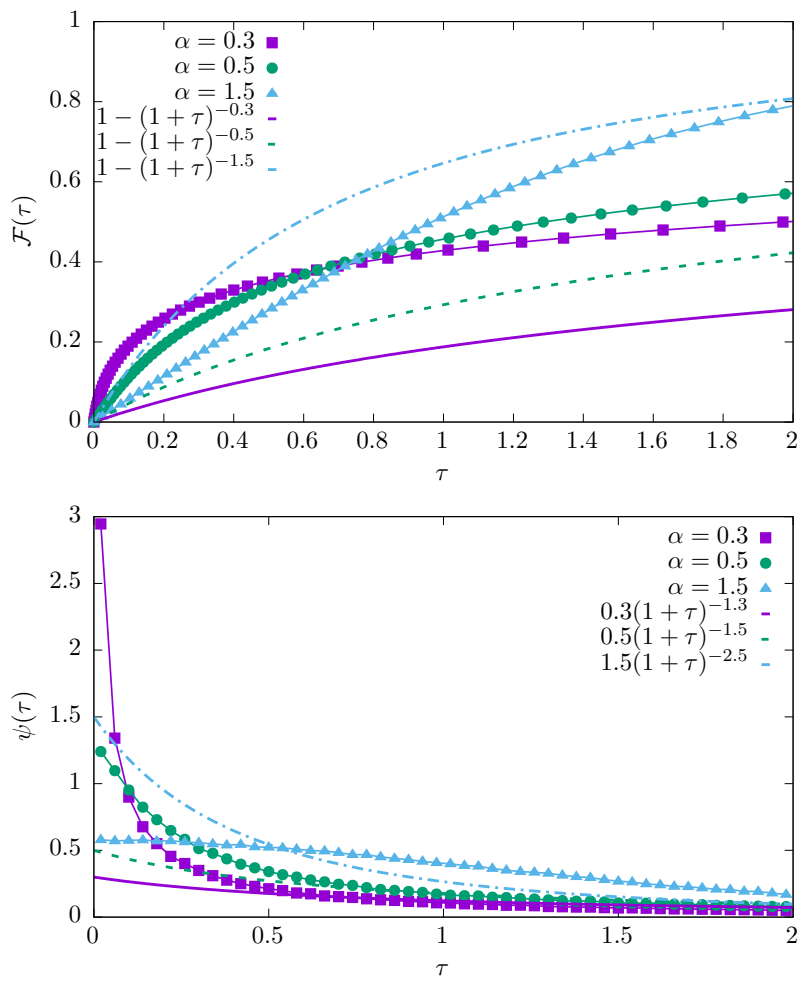

Figure 14. The associated cumulative distributions $\mathcal{F}(\tau)$ (top panel) and probability densities $\psi(\tau)$ (bottom panel) for $\alpha$-stable and Pareto densities with $\alpha=0.3,0.5,1.5$. 
velocity prevents immediate velocity reversal, see bottom panel of figure 12 where the secondary peaks in $p(x)$ and $p(v)$ are absent. Nevertheless, the possibility of reversals is manifested by the extended widths of the primary (without velocity reversals) peaks.

Finally, in order to verify the hypothesis of the immediate reversal we used a different waiting time distribution, namely, the Pareto density

$$
p(\tau)=\alpha(1+\tau)^{-(\alpha+1)},
$$

with $\alpha>0$. The density 30 has the same power-law asymptotics as the applied $\alpha$ stable density, see Eq. (7). Figure 13 presents the same results as figure 12 for the Pareto density (30). It is clearly visible that for the Pareto density of wating times there are no secondary peaks, corresponding to the immediate velocity reversal, compare top panels of figures 12 and 13 . Additionally, the Pareto distribution also changes the structure of the peaks in the absence of an initial velocity, that is for $v(0)=0$, see bottom panels of figures 12 and 13, when they become narrow in comparison to the case of an $\alpha$-stable waiting time distribution. Comparison of figures 12 and 13 attributes the structure of the peaks and the possibility of immediate reversal to the central $(\tau \approx 0)$ part of the waiting time distributions. This is further analysed in figure 14 in which probability densities $\psi(\tau)$ and cumulative densities

$$
\mathcal{F}(\tau)=\operatorname{Prob}\left(\tau^{\prime} \leqslant \tau\right)=\int_{0}^{\tau} \psi\left(\tau^{\prime}\right) d \tau^{\prime}
$$

corresponding to $\alpha$-stable and Pareto waiting time distributions are presented. Figure 14 clearly demonstrates that for small values of $\alpha$ the central parts $(\tau \approx 0)$ of the waiting time distribution contain more probability mass for an $\alpha$-stable density than for a Pareto distribution. In turn, this indicates that the central part of waiting time distribution controls the probability of immediate velocity reversal.

\section{Summary and Conclusions}

Lévy walks are continuous time random walks with a spatiotemporal coupling between jump lengths and waiting times. This effects that long jumps are penalised by long corresponding waiting times, and thus the emerging mean squared displacement is always finite, in contrast to uncoupled Lévy flights. For the latter the diverging variance of the long-tailed jump length distribution translates into the divergence of the mean squared displacement. Most frequently the simplest spatiotemporal coupling using a constant speed is used. In the velocity model [28] this means that the waiting times determine the time between velocity changes. In such a scenario no external forces are considered such that the absolute value of the velocity, the speed $|v|$, is always constant. Lévy walks are conservative in the sense that their kinetic energy is constant.

Here, we have studied an extension of Lévy walk processes to cases in which external forces influence the motion of the test particle. As in the standard Lévy walk scenario we assume that the random walker deterministically continues its motion for a random time. At the renewal time, a velocity reversal occurs. We call this a hard velocity reversal, as 


\begin{tabular}{|c|c|c|c|c|c|}
\hline & $n=1$ & & $1<n$ & $\infty$ & $n=\infty$ \\
\hline \multirow{2}{*}{$\begin{array}{l}p(\tau) \propto \tau^{-\alpha-1} \\
\quad(0<\alpha<1)\end{array}$} & $p(v)$ & uniform & $p(v)$ & U-shaped & $\begin{array}{l}p(v)=\frac{1}{2}[\delta(v- \\
\left.\left.v_{0}\right)+\delta\left(v+v_{0}\right)\right]\end{array}$ \\
\hline & $\begin{array}{l}p(x) \\
\text { slowly } \\
\text { peaks } \\
\text { PDFs }\end{array}$ & $\begin{array}{l}\text { U-shaped } \\
\text { decaying } \\
\text { in both }\end{array}$ & $\begin{array}{l}p(x) \\
\text { slowly } \\
\text { peaks } \\
\text { PDFs }\end{array}$ & $\begin{array}{l}\text { U-shaped } \\
\text { decaying } \\
\text { in both }\end{array}$ & $\begin{array}{lr}p(x) & \text { uniform } \\
\text { slowly } & \text { decaying } \\
\text { peaks in } p(x) \\
\text { only }\end{array}$ \\
\hline \multirow{2}{*}{$\begin{array}{r}p(\tau) \propto \tau^{-\alpha-1} \\
\quad(1<\alpha \leqslant 2)\end{array}$} & $p(v)$ & uniform & $p(v)$ & U-shaped & $\begin{array}{l}p(v)=\frac{1}{2}[\delta(v- \\
\left.\left.v_{0}\right)+\delta\left(v+v_{0}\right)\right]\end{array}$ \\
\hline & $\begin{array}{l}p(x) \\
\text { fast } \\
\text { peaks } \\
\text { PDFs }\end{array}$ & $\begin{array}{l}\text { U-shaped } \\
\text { decaying } \\
\text { in both }\end{array}$ & $\begin{array}{l}p(x) \\
\text { fast } \\
\text { peaks } \\
\text { PDFs }\end{array}$ & $\begin{array}{l}\text { U-shaped } \\
\text { decaying } \\
\text { in both }\end{array}$ & $\begin{array}{ll}p(x) & \text { uniform } \\
\text { fast decaying } \\
\text { peaks in both } \\
\text { PDFs }\end{array}$ \\
\hline
\end{tabular}

Table 3. Probability densities for conservative random walks in single well potentials for different values of the power exponent $n$ of the external potential as well as for waiting time densities of power law form with diverging $(0<\alpha<1)$ and finite $(1<\alpha \leqslant 2)$ mean waiting time. For exponential waiting times the same behaviour is found as for the case $1<\alpha \leqslant 2$.

typically the velocity of the particle at a random instant of time is finite. In contrast to the standard Lévy walk model, however, the speed is no longer constant. In the conservative random walk model adopted here the total energy of the particle consisting of the potential energy in the external force field and its kinetic energy, is supposed to be a constant. This necessarily requires that the velocity changes deterministically and perpetually, according to Newton's second law of motion. This scenario then also leads to soft velocity reversals at points of maximal distance from the centre of the potential, when the potential energy assumes its maximum and the particle velocity is zero. In this conservative random walk model the sole source of stochasticity is in the velocity reversal, similar to the standard Lévy walk model. Moreover, we demonstrated that the relaxation dynamics of the system visible in the decay of the envelope of $|\langle x(t)\rangle|$ is of power-law form.

From analytical calculations and numerical results we calculated the velocity and position distributions for Lévy walks in single well potentials of the form $V(x)=|x|^{n} / n$ type with $n=1,2,4$, and $\infty$. The exact solutions we obtained agree perfectly with the shapes of the densities estimated from Monte Carlo simulations of the underlying 
motion. For waiting times with diverging mean (with power law exponent $0<\alpha<1$ ) the densities are decorated with distinct peaks, which slowly decay as function of time. Regardless of the potential type, the peak height decays as a power-law function of time, with the decay rate depending on the scaling exponent $\alpha$, see table A1 and Fig. A3. For more details see the appendix. These peaks quickly disappear when the mean waiting time is finite, corresponding to the cases of power law waiting time distributions with $1<\alpha \leqslant 2$ or exponential waiting time distributions. When the motion occurs in symmetric single well potentials with $n=2$ or 4 , the distribution of both velocity and position are U-shaped. For the rectangular potential well both distributions are flat. In the intermediate case $n=1$ the position distribution is U-shaped while the velocity distribution is flat. The central properties for the different relevant cases are summarised in table 3 ,

The studied extension of the Lévy walk scenario provides a possibility to verify which details of the dynamics are sensitive to the tails and which are sensitive to the central parts of the waiting time distributions. Namely, the tail of the waiting time distribution controls the rate of convergence to the stationary state. Therefore, for $\alpha<1$, it is responsible for the appearance of the slowly vanishing peaks decorating the stationary states. The width of the central part of the waiting time distribution around $\tau \approx 0$ is responsible for the appearance of secondary peaks in the position and velocity distributions which originate in the significant possibility of immediate velocity reversal. Consequently, there are two control mechanisms that allow one to eliminate secondary peaks. The first possibility is to use $v(0)=0$ initial condition. The second option is to use a narrower waiting time distribution. The former scenario diminishes secondary peaks but still preserves non-zero width of primary peaks. The latter scenario not only eliminates secondary peaks but also reduces the width of the primary peaks. We note again that regardless of the existence or divergence of the mean waiting time, in the long time limit the peaks disappear.

The studied model provides a possible generalisation of the Lévy walk model which accounts for external forces. Within this model, in analogy to free Lévy walks the system is conservative. The system energy consists of kinetic and potential energy. The studied model can be contrasted with other, non-conservative extensions of Lévy walks [48, 49, 50, 51].

\section{Acknowledgments}

This project has been supported in part by the Polish National Science Center grant (2014/13/B/ST2/02014) and by grant ME 1535/6-1 and ME 1535/7-1 from Deutsche Forschungsgemeinschaft. RM thanks the Foundation for Polish Science for support within an Alexander von Humboldt Polish Honorary Research Scholarship. Computer simulations were performed at the Academic Computer Center Cyfronet, Akademia Górniczo-Hutnicza (Kraków, Poland) under CPU grant DynStoch. 

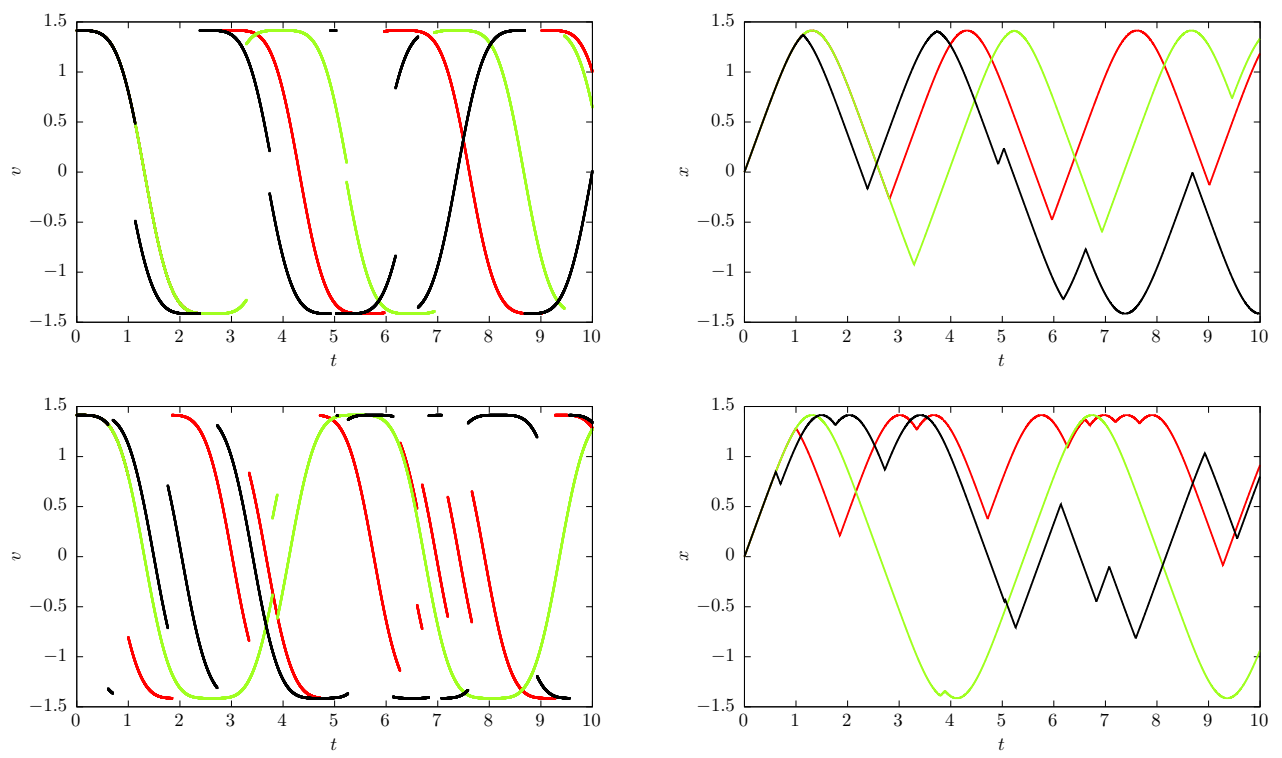

Figure A1. Three sample trajectories for $\alpha=0.5$ (top panel) and $\alpha=1.5$ (bottom panel) for $n=4$ with identical initial conditions. In the left column we show the velocity $v(t)$, the right column depicts the position $x(t)$. The plots evidence the randomisation of sample paths due to hard velocity reversals.

\section{Appendix A. Further details on the conservative Lévy walk dynamics}

Figure A1 complements figure 4 and investigates the role of randomness in the system dynamics. In contrast to figure 4. it presents three sample trajectories for $\alpha=0.5$ (top panel) and $\alpha=1.5$ (bottom panel) for $n=4$ corresponding to the same initial conditions. The trajectories start with identical initial conditions but soon become randomised due to hard velocity reversals. Figure A1 demonstrates that with increasing value of $\alpha$ a larger number of hard velocity reversal is observed.

In order to quantify how the stationary state is reached we analyse dependence of $\langle x(t)\rangle$ in more detail. From $\langle x(t)\rangle$ shown in figure 7 the absolute value $|\langle x(t)\rangle|$ was calculated. We then determined the envelope $|\langle x(t)\rangle|_{\mathrm{e}}$ corresponding to the local maxima of $|\langle x(t)\rangle|$. These envelopes were then fitted by a power-law. Figure A2 presents the envelope $|\langle x(t)\rangle|_{\mathrm{e}}$ for $\alpha=0.5$ (top panel) and $\alpha=1.5$ (bottom panel) for $n=1,2,4$. From figure A2 it is clearly visible that for $\alpha=0.5$ the envelope $|\langle x(t)\rangle|_{\mathrm{e}}$ decays as a power-law with exponent $=-0.4$, which is close to the value 0.5 of $\alpha$, independent of the steepness of the potential characterised by $n$. For $\alpha=1.5$, in contrast, the power-law decay is significantly faster with an exponent depending on $n$.

Finally, in order to measure the convergence rate to the stationary state we extracted the relative heights $h(t)$ of the deterministic peaks decorating the probability densities. From this data we constructed time series showing peak heights measured from the background given by the density without peaks. These time series were then used to fit the power-law decay

$$
h_{v}(t)=\beta_{v} \times t^{\alpha_{v}}
$$



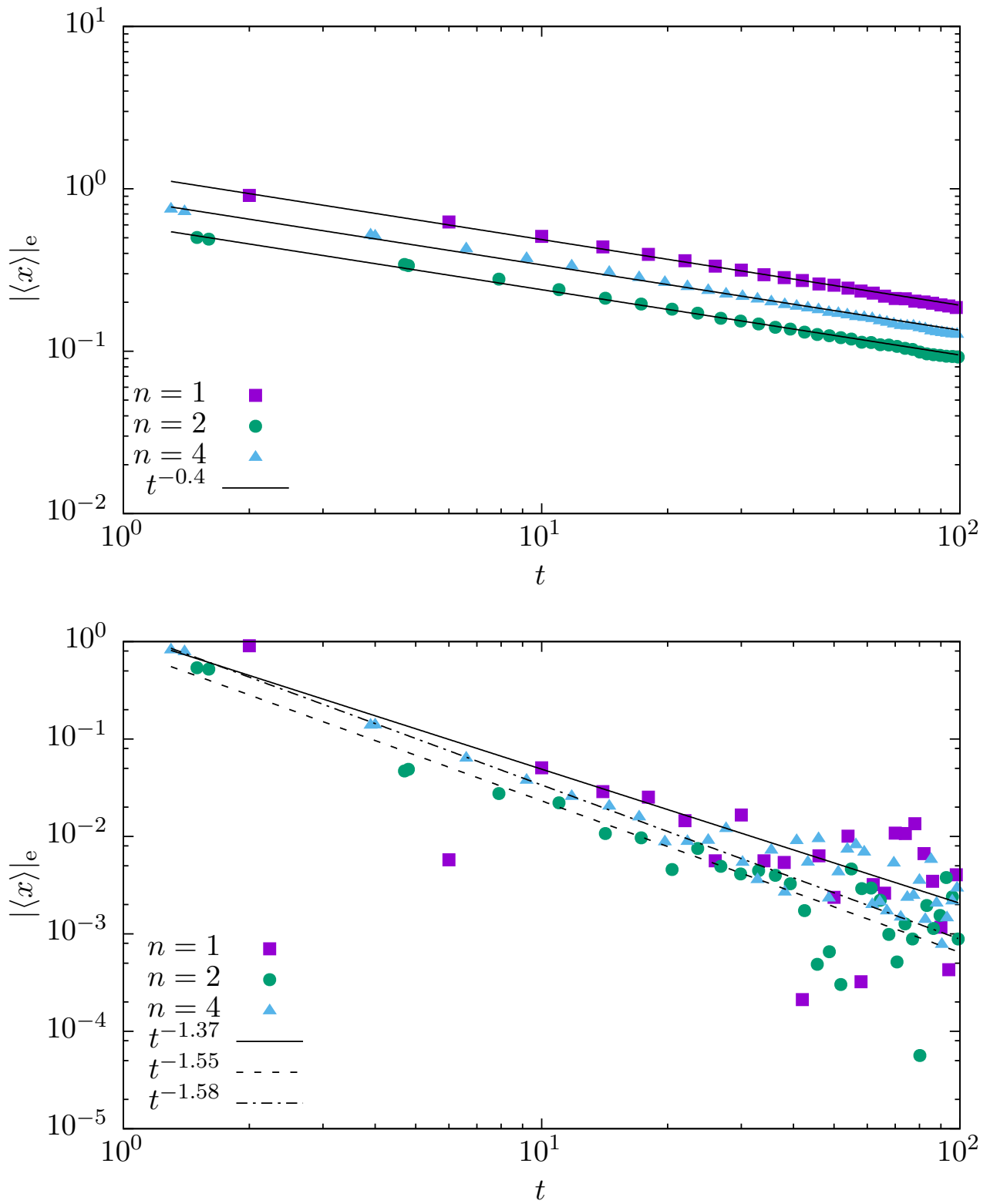

Figure A2. Decay of the envelope $|\langle x(t)\rangle|_{\mathrm{e}}$ representing the local maxima of $|\langle x(t)\rangle|$, for $\alpha=0.5$ (top panel) and $\alpha=1.5$ (bottom panel). The scaling exponents for the apparent power-law behaviour are indicated in the panels.

and

$$
h_{x}(t)=\beta_{x} \times t^{\alpha_{x}} .
$$

Figure A3 presents results for the harmonic potential. Empty symbols show the peak heights in the velocity distribution while full symbols refer to the position distribution. In both distributions the dependence of the peak height is practically identical. For $\alpha<2$, the decay rate is of power-law type. The scaling exponent characterising the decay is very sensitive to the value of the stability index $\alpha$, see table A1. For $\alpha<1$ the decay is very slow, consequently peaks decorating the time dependent densities take a very long time to disappear. In contrast, for $1<\alpha<2$ the decay still seems 


\begin{tabular}{c|c||c||c}
$n$ & $\alpha$ & $\alpha_{v} \pm \Delta \alpha_{v}$ & $\alpha_{x} \pm \Delta \alpha_{x}$ \\
\hline 1 & 0.3 & $-0.237 \pm 0.001$ & $-0.238 \pm 0.001$ \\
1 & 0.5 & $-0.441 \pm 0.001$ & $-0.441 \pm 0.001$ \\
1 & 1 & $-0.929 \pm 0.003$ & $-0.936 \pm 0.003$ \\
1 & 1.5 & $-1.684 \pm 0.009$ & $-1.647 \pm 0.017$ \\
\hline 2 & 0.3 & $-0.239 \pm 0.001$ & $-0.240 \pm 0.001$ \\
2 & 0.5 & $-0.444 \pm 0.001$ & $-0.445 \pm 0.001$ \\
2 & 1 & $-0.914 \pm 0.003$ & $-0.913 \pm 0.003$ \\
2 & 1.5 & $-1.561 \pm 0.013$ & $-1.573 \pm 0.012$ \\
\hline 4 & 0.3 & $-0.238 \pm 0.001$ & $-0.243 \pm 0.001$ \\
4 & 0.5 & $-0.439 \pm 0.001$ & $-0.443 \pm 0.001$ \\
4 & 1 & $-0.898 \pm 0.004$ & $-0.901 \pm 0.003$ \\
4 & 1.5 & $-1.394 \pm 0.027$ & $-1.621 \pm 0.007$ \\
\hline 6 & 0.3 & $-0.223 \pm 0.002$ & $-0.231 \pm 0.001$ \\
6 & 0.5 & $-0.440 \pm 0.002$ & $-0.442 \pm 0.001$ \\
6 & 1 & $-0.905 \pm 0.004$ & $-0.921 \pm 0.003$ \\
6 & 1.5 & $-1.298 \pm 0.041$ & $-1.632 \pm 0.009$
\end{tabular}

Table A1. Exponents characterising decay of peaks decorating velocity $\left(\alpha_{v}\right)$ and positions $\left(\alpha_{x}\right)$ distributions as a function of the stability index $\alpha$ and the potential steepness $n$.

to be of power-law type but it is fast enough to diminish the peaks practically quite fast. Therefore, on the one hand, regardless of value of $\alpha$ there is the same mechanism responsible for the decay of memory about initial conditions. On the other hand, the observed time scales strongly depend on $\alpha$. Interestingly, the exponents describing the decay are weakly sensitive to the potential type, see table A1.

\section{References}

[1] R. Brown, Phil. Mag. 2 4, 161 (1828)

[2] M. Gouy, J. Phys. Theor. Appl. 7, 561 (1888)

[3] J. Perrin, Compt. Rend. 146, 967 (1908)

[4] I. Nordlund, Z. Phys. Chem 87, 40 (1914)

[5] E. Kappler, Ann. Phys. (Leipzig) 403, 233 (1931)

[6] M. Von Smoluchowski, Ann. Phys. 21, 756 (1906)

[7] A. Einstein, Ann. Phys. 17, 549 (1905)

[8] P. Langevin, C. R. Acad. Sci. Paris 146, 530 (1908)

[9] D.R. Cox, H.D. Miller, The theory of stochastic processes (Chapman and Hall, London, 1965)

[10] H. Risken, The Fokker-Planck equation. Methods of solution and application (Springer Verlag, Berlin, 1984)

[11] G. Samorodnitsky, M.S. Taqqu, Stable non-Gaussian random processes: Stochastic models with infinite variance (Chapman and Hall, New York, 1994) 


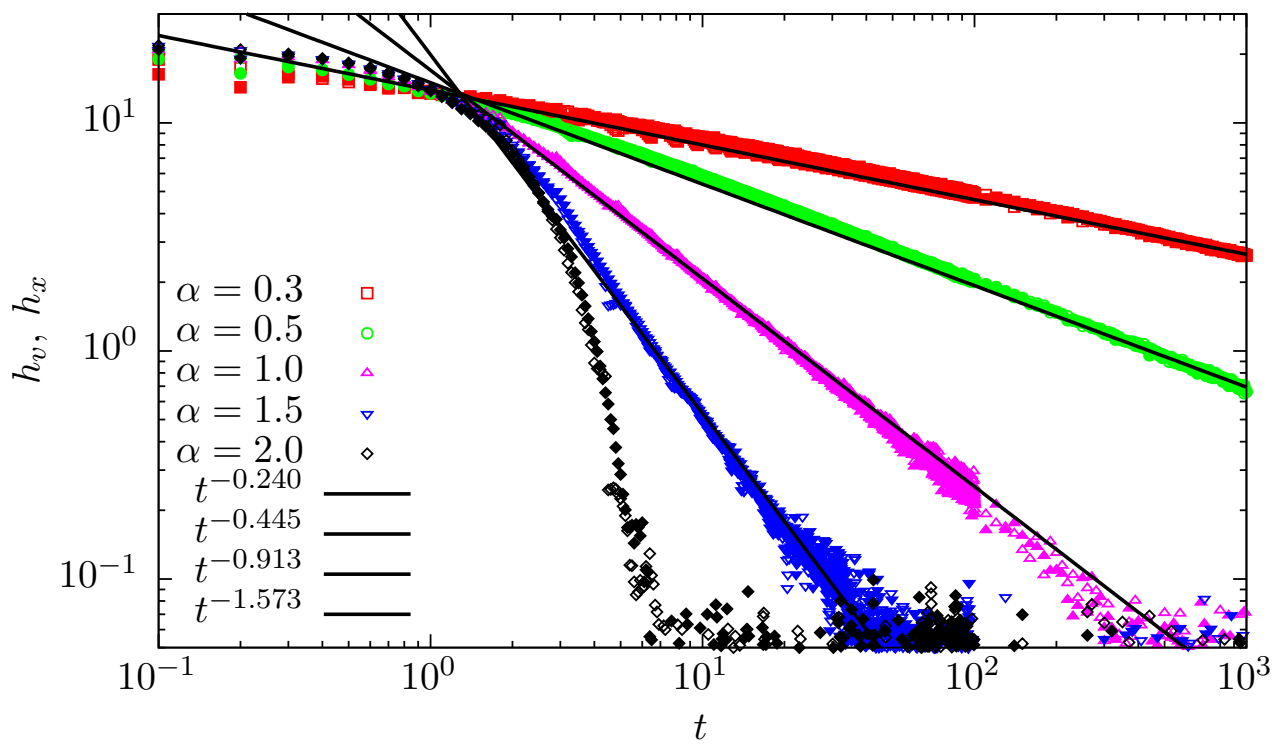

Figure A3. Decay of peak height $h_{v}(t)$ decorating velocity (empty symbols) and position $h_{x}(t)$ (full symbols) distributions along with fitted slopes, see table A1.

[12] A. Janicki, A. Weron, Simulation and chaotic behavior of $\alpha$-stable stochastic processes (Marcel Dekker, New York, 1994)

[13] W. Horsthemke, R. Lefever, Noise-inducted transitions. Theory and applications in physics, chemistry, and biology (Springer Verlag, Berlin, 1984)

[14] M.F. Shlesinger, G.M. Zaslavsky, J. Frisch, eds., Lévy flights and related topics in physics (Springer Verlag, Berlin, 1995)

[15] H.C. Fogedby, Phys. Rev. Lett 73, 2517 (1994)

[16] S. Jespersen, R. Metzler, H.C. Fogedby, Phys. Rev. E 59, 2736 (1999)

[17] G.M. Viswanathan, M.G. Da Luz, E.P. Raposo, H.E. Stanley, The physics of foraging: An introduction to random searches and biological encounters (Cambridge University Press, Cambridge, 2011)

[18] V.V. Palyulin, A.V. Chechkin, R. Metzler, Proc. Natl. Acad. Sci. U.S.A. 111, 2931 (2014)

[19] C. Cattaneo, Atti Sem. Mat. Fis. Univ. Modena 3, 83 (1948)

[20] D. Jou, J. Casas-Vázquez, G. Lebon, Extended Irreversible Thermodynamics (Springer Verlag, Berlin, 1996)

[21] P.C. De Jagher, Physica A 101, 629 (1980)

[22] E.W. Montroll, M.F. Shlesinger, On the wonderful world of random walks, in Lévy processes: Theory and applications, edited by J.L. Lebowitz, E.W. Montroll (North Holland, Amsterdam, 1984), pp. 1-121

[23] R. Metzler, J. Klafter, Phys. Rep. 339, 1 (2000)

[24] R. Metzler, Phys. Rev. E 62, 6233 (2000).

[25] M.F. Shlesinger, J. Klafter, Y.M. Wong, J. Stat. Phys. 27, 499 (1982)

[26] M.F. Shlesinger, J. Klafter, Lévy walks versus Lévy flights, in On growth and form: Fractal and non-fractal patterns in physics, edited by H.E. Stanley, N. Ostrowsky (Springer Verlag, Berlin, 1986), p. 279

[27] V. Zaburdaev, S. Denisov, J. Klafter, Rev. Mod. Phys. 87, 483 (2015)

[28] G. Zumofen, J. Klafter, Physica D 69, 436 (1993)

[29] T. Geisel, S. Thomae, Phys. Rev. Lett. 52, 1936 (1984)

[30] T.H. Solomon, E.R. Weeks, H.L. Swinney, Phys. Rev. Lett. 71, 3975 (1993)

[31] D.A. Kessler, E. Barkai, Phys. Rev. Lett. 108, 230602 (2012) 
[32] M.A. Lomholt, T. Koren, R. Metzler, J. Klafter, Proc. Natl. Acad. Sci. USA 105, 11055 (2008)

[33] M.S. Song, H.C. Moon, J.H. Jeon, H.Y. Park, Nature Comm. 9, 344 (2018)

[34] K. Chen, B. Wang, S. Granick, Nature Mat. 14, 589 (2015)

[35] R. Metzler, J.H. Jeon, A.G. Cherstvy, E. Barkai, Phys. Chem. Chem. Phys. 16, 24128 (2014)

[36] A. Godec, R. Metzler, Phys. Rev. Lett. 110, 020603 (2013)

[37] A. Godec, R. Metzler, Phys. Rev. E 88, 012116 (2013)

[38] D. Froemberg, E. Barkai, Eur. Phys. J. B 86, 331 (2013)

[39] D. Froemberg, M. Schmiedeberg, E. Barkai, V. Zaburdaev, Phys. Rev. E 91, 022131 (2015)

[40] F. Jülicher, A. Ajdari, and J. Prost, Rev. Mod. Phys. 69, 1269 (1997).

[41] I. Goychuk, V. O. Kharchenko, and R. Metzler, Phys. Chem. Chem. Phys. 16, 16524 (2014).

[42] L.D. Landau, E.M. Lifshits, Theoretical Physics: Mechanics (Pergamon Press, Oxford, 1988)

[43] B. Hughes, Random walks and random environments (Clarendon Press, Oxford, 1995)

[44] D. Campos, E. Abad, V. Méndez, S.B. Yuste, K. Lindenberg, Phys. Rev. E 91, 052115 (2015)

[45] B. Dybiec, E. Gudowska-Nowak, E. Barkai, A.A. Dubkov, Phys. Rev. E 95, 052102 (2017)

[46] A Vezzani, E. Barkai and R. Burioni, The Single Big Jump Principle in Physical Modelling arXiv:1804.02932 (2018)

[47] W.H. Press, S.A. Teukolsky, W.T. Vetterling, B.P. Flannery, Numerical recipes. The art of scientific computing (Cambridge University Press, Cambridge, 1992)

[48] G. Gradenigo, A. Sarracino, D. Villamaina, A. Vulpiani, J. Stat. Mech. 2012, L06001 (2012)

[49] R. Burioni, G. Gradenigo, A. Sarracino, A. Vezzani, A. Vulpiani, J. Stat. Mech. 2013, P09022 (2013)

[50] A. Dechant, S.T. Shafier, D.A. Kessler, E. Barkai, Phys. Rev. E 94, 022151 (2016)

[51] A. Dechant, D.A. Kessler, E. Barkai, Phys. Rev. Lett. 115, 173006 (2015) 